\title{
Knowledge Representation and Management: Towards an Integration of a Semantic Web in Daily Health Practice
}

\author{
N. Griffon ${ }^{1,3}$, J. Charlet ${ }^{2,3}$, SJ. Darmoni ${ }^{13}$, Section Editors for the IMIA Yearbook 2013, Sec- \\ tion on Knowledge Representation and Management \\ 1 CISMeF-TIBS-LITIS EA 4108, Rouen University Hospital, Rouen, France \\ ${ }^{2}$ AP-HP, Dept. of Clinical Research and Development, Paris, France \\ ${ }^{3}$ INSERM UMRS 872 eq. 20, CRC, Paris, France
}

\begin{abstract}
Summary
Objective: To summarize the best papers in the field of

Knowledge Representation and Management (KRM).

Methods: A synopsis of the four selected articles for the IMIA

Yearbook 2013 KRM section is provided, as well as highlights of

current KRM trends, in particular, of the semantic web in daily

health practice. The manual selection was performed in three

stages: first a set of 3,106 articles, then a second set of 86

articles followed by a third set of 15 articles, and finally the last

set of four chosen articles.

Results: Among the four selected articles (see Table 1), one focuses on knowledge engineering to prevent adverse drug events; the objective of the second is to propose mappings between clinical archetypes and SNOMED CT in the context of clinical practice; the third presents an ontology to create a question-answering system; the fourth describes a biomonitoring network based on semantic web technologies.

Conclusion: These four articles clearly indicate that the health semantic web has become a part of daily practice of health professionals since 2012. In the review of the second set of 86 articles, the same topics included in the previous IMIA yearbook remain active research fields: Knowledge extraction, automatic indexing, information retrieval, natural language processing, management of health terminologies and ontologies.
\end{abstract}

\section{Keywords}

Semantic web; knowledge management; abstracting and indexing as a topic; vocabulary, controlled; biomonitoring;

Yearb Med Inform 2013:155-8

\section{Introduction}

"Evidence based medicine is the theme of the 2013 Yearbook of Medical Informatics. In the last five years, the intersection between evidenced-based medicine and biomedical informatics, which can be termed "evidence-based biomedical informatics" is steadily growing. In this synopsis, we review how evidence-based biomedical informatics has impacted on the Knowledge Representation and Management (KRM) section of the 2013 Yearbook.

\section{Best Paper Selection}

For the KRM section of the IMIA Yearbook 2013, four papers were selected based on the following review process [1]: (1) two queries were launched in PubMed and Web of Science as these two bibliographic databases were considered by consensus of IMIA Yearbook section editors to be the most relevant. These two queries are available at the following URL: http:// www.chu-rouen.fr/documed/yearbook12/ $\mathrm{krm} . \mathrm{html}$ and in order for the results to be replicated need to be cut and pasted respectively in PubMed and in Web of Science. The main MeSH terms used in the PubMed query were: ("Knowledge"[MH] OR "Knowledge bases" [MH] OR "Knowledge management"[MH] OR "Abstracting and indexing as topic"[MH] OR "Vocabulary, controlled"' $[\mathrm{MH}])$. A number of terms were added to cover more articles using [TIAB]
(Title or Abstract) metadata. A total of 3,506 unique articles were obtained from these two queries. One section editor (NG) reviewed each article based on the title and abstract and selected 86 papers. The main selection criterion was the adequacy to the theme section. These 86 papers were then reviewed by the three section editors. Fifteen articles were selected by consensus. Then, each of these 15 articles was read and rated by the same three experts. The list was subsequently discussed by all section editors and the two co-editors during a one day conference meeting. The top four articles were finally selected.

These four papers are shown in Table 1 and are summarized in the appendix of this synopsis.

Koutkias et al. [2] proposed a contextualized knowledge-based framework to prevent adverse drug events via a specific computer-aided decision support system. This study was developed and granted as part of the PSIP project [3], and funded by the European Union $7^{\text {th }}$ Framework Program. Meizoso García et al. [4] developed and evaluated mappings between clinical archetypes and SNOMED CT in the context of clinical practice. Several Natural Language Processing (NLP) tools were used to implement these mappings. Patrick and Li [5] developed a very original paper on building an ontology to create a clinical question-answering (QA) system based on clinical notes. Teodoro et al. [6] evaluated and have described a biomonitoring network based on semantic web technologies. This ARTEMIS network provides a real-time antimicrobial 
Table 1 Best paper selection of articles for the IMIA Yearbook of Medical Informatics 2013 in the sections 'Knowledge Represenation and Management. The articles are listed in alphabetical order of the first author's surname.

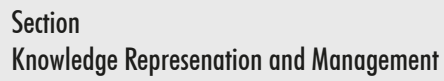

- Koutkias V, Kilintzis V, Stalidis G, Lazou K, Niès J, Durand-Texte L, McNair P, Beuscart R, Maglaveras N. Knowledge engineering for adverse drug event prevention: on the design and development of a uniform, contextualized and sustainable knowledge-based framework. J Biomed Inform 2012 Jun;45(3):495-506.

- Meizoso García M, Iglesias Allones JL, Martínez Hernández D, Taboada Iglesias MJ. Semantic similarity-based alignment between clinical archetypes and SNOMED CT: an application to observations. Int J Med Inform 2012 Aug;81(8):566-78.

- Patrick J, Li M. An ontology for clinical questions about the contents of patient notes. J Biomed Inform 2012 Apr; 45(2):292-306.

- Teodoro D, Pasche E, Gobeill J, Emonet S, Ruch P, Lovis C. Building a transnational biosurveillance network using semantic web technologies: requirements, design, and preliminary evaluation. J Med Internet Res 2012 May 29;14(3):e73.

resistance monitoring system, and is already piloted in several hospitals. This work was developed during the DebugIT project [7], and also funded by the European Union $7^{\text {th }}$ Framework Program.

When reviewing the broader second set of 86 selected articles, the same topics included in the previous IMIA yearbook [8] were identified to still be active research topics by the three section editors of the KRM i.e.: knowledge extraction, automatic indexing, information retrieval, natural language processing, management of health terminologies and ontologies using semantic web technologies. A particular hot topic was the mapping methods and techniques between archetypes, interface terminologies as well as reference terminologies and ontologies. These mappings are mandatory basic tools to create evidence-based biomedical informatics. This is particularly true for computer-aided decision systems.

\section{Conclusion and Outlook}

The four selected articles for the KRM section clearly confirm that the health semantic web is now an integral part of daily practice of health professionals in 2012. Several specialized conferences already exist: Knowledge Representation in Medicine (KR-Med) [9] organized by the AMIA working group Knowledge Representation and Semantics [10], the international conference on biomedical ontology (ICBO) [11], and Semantic Web Applications and Tools for
Life Sciences (SWAT4LS) [12]. An interest group has also been created on this topic within W3C: the Semantic Web Health Care and Life Sciences Interest Group [13]. In 2011, a network of excellence was funded by the European Union on the same topic: Semantic Interoperability for Health Network (SemanticHealthNet) [14]. Its objective is to develop a scalable and sustainable pan-European organizational and governance process for the semantic interoperability of clinical and biomedical knowledge, and to help ensure that electronic health record (EHR) systems are optimized for patient care, public health and clinical research across healthcare systems and institutions [14]. Health terminologies and ontologies (HTO) in health information systems are spreading in English via BioPortal [15] and other languages, in particular in French with more than 30 HTO available via a cross-lingual portal (URL: www.hetop.eu) [16]. Informatics for Integrating Biology \& the Bedside (I2B2) [17] with its *omics extension TRANSMART (genomics, proteomics, metabolomics, etc) is an operational platform to enable clinical researchers to perform information retrieval in EHRs. I2B2/Transmart is becoming a "de facto" standard used by a considerable number of university hospitals around the world to practice translational medicine by health professionals.

\section{Acknowledgments}

The authors thank Martina Hutter for her consistent support and Richard Medeiros, Rouen University Hospital Medical Editor, for editing this manuscript.

\section{References}

1. Lamy JB, Seroussi B, Griffon N, Kerdelhue G, Jaulent MC, Bouaud J. Formalizing literature search and review strategy to select candidate best papers for the IMIA Yearbook. Submitted to AMIA 2013.

2. Koutkias V, Kilintzis V, Stalidis G, Lazou K, Niès $\mathrm{J}$, et al. Knowledge engineering for adverse drug event prevention: on the design and development of a uniform, contextualized and sustainable knowledge-based framework. J Biomed Inform 2012 Jun;45(3):495-506.

3. Patient Safety Through Intelligent Procedures in Medication (PSIP). URL: http://www.psip-project. eu/ (Accessed April, 30 2012).

4. Meizoso García M, Iglesias Allones JL, Martínez Hernández D, Taboada Iglesias MJ. Semantic similarity-based alignment between clinical archetypes and SNOMED CT: an application to observations. Int J Med Inform 2012 Aug;81(8):566-78.

5. Patrick J, Li M. An ontology for clinical questions about the contents of patient notes. J Biomed Inform. 2012 Apr;45(2):292-306.

6. Teodoro D, Pasche E, Gobeill J, Emonet S, Ruch $\mathrm{P}$, et al. Building a transnational biosurveillance network using semantic web technologies: requirements, design, and preliminary evaluation. J Med Internet Res 2012 May 29;14(3):e73.

7. Detecting and Eliminating Bacteria Using Information Technology (DebugIT). URL: http://www. debugiteu/ (Accessed April, 30 2012).

8. Rassinoux AM. Knowledge Representation and Management: Towards Patient Health Self-management.Yearb Med Inform 2012;7(1):126-9.

9. Knowledge Representation in Medicine (KR-Med). URL: kr-med.org/ (Accessed April, 30 2012).

10. AMIA working group Knowledge Representation and Semantics. URL: http://www.amia.org/ programs/working-groups/knowledge-representation-and-semantics (Accessed April, 30 2012).

11. International Conference on Biomedical Ontology (ICBO). http://kr-med.org/icbofois2012/icbo.htm (Accessed April, 30 2012).

12. Semantic Web Applications and Tools for Life Sciences (SWAT4LS). URL: http://www.swat4ls. org/ (Accessed April, 30 2012).

13. Semantic Web Health Care and Life Sciences Interest Group. URL: http://www.w3.org/blog/ hcls/ (Accessed April, 29 2012).

14. Semantic Interoperability for Health Network. URL: http://www.semantichealthnet.eu/ (Accessed April, 29 2012).

15. Whetzel PL, Noy NF, Shah NH, Alexander PR, Nyulas C, Tudorache T, et al. BioPortal: enhanced functionality via new Web services from the National Center for Biomedical Ontology to access and use ontologies in software applications. Nucleic Acids Res 2011 Jul;39 (Web Server issue):W541-5.

16. Grosjean J, Merabti T, Griffon N, Dahamna B, Darmoni SJ. Teaching medicine with a terminology/ontology portal. Stud Health Technol Inform 2012;180:949-53.

17. Informatics for Integrating Biology \& the Bedside (I2B2). URL: https://www.i2b2.org (Accessed April, 30 2012). 
Correspondence to:

Prof. SJ. Darmoni, MD, PhD.

Rouen University Hospital

Department of BioMedical Informatics

1 rue de Gérmont

76031 Rouen Cedex, France

Tel: +33(0)232 888829

Fax: +33(0)232888909

E-mail: stefan.darmoni@chu-rouen.fr

Appendix: Content Summaries of Selected Best Papers for the IMIA Yearbook 2013, Section Knowledge Representation and Management ${ }^{\top}$

\author{
Koutkias V, Kilintzis V, Stalidis G, Lazou K, \\ Niès J, Durand-Texte L, McNair P, Beuscart \\ $\mathrm{R}$, Maglaveras $\mathrm{N}$
}

Knowledge engineering for adverse drug event prevention: on the design and development of a uniform, contextualized and sustainable knowledge-based framework

\section{J Biomed Inform 2012 Jun;45(3):495-506}

This article presents a sustainable knowledge-based framework to support adverse drug event prevention via Clinical Decision Support Systems. The framework was developed during the Patient Safety Through Intelligent Procedures in Medication European (PSIP) project. The methodology involved the systematic analysis and formalization of the knowledge sources elaborated in the scope of this work, for which an application-specific knowledge model had been defined. The entire architectural framework was specified and implemented by adopting Computer Interpretable Guidelines as the knowledge engineering formalism for its construction. The framework integrates diverse and dynamic knowledge sources in the form of rule-based adverse drug event signals, all

\footnotetext{
The complete papers can be accessed in the Yearbook's full electronic version, provided that the article is freely accessible or that your institution has access to the respective journal.
}

under a uniform Knowledge Base structure, according to the defined knowledge model. Equally important, it employs the means to contextualize the encapsulated knowledge, in order to provide appropriate support considering the specific local environment (hospital, medical department, language, etc.), as well as the mechanisms for knowledge querying, inference, sharing, and management.

Therefore, in this paper, the authors insist on the results obtained: implementation performance and validation aspects that highlight its applicability and virtue in medication safety.

\section{MeizosoGarcía M, Iglesias Allones JL, Martínez Hernández D, Taboada Iglesias MJ}

Semantic similarity-based alignment between clinical archetypes and SNOMED CT: an application to observations

\section{Int J Med Inform 2012 Aug;81(8):566-78}

Clinical data models, such as OpenEHR Archetypes, define data structures that are agreed upon by experts to ensure the accuracy of health information. In addition, they provide an option to normalize clinical data by means of binding terms used in the model definition in standard medical vocabularies. Nevertheless, the effort needed to establish the association between archetype terms and standard terminology concepts is considerable. Therefore, the purpose of this study was to provide an automated approach to bind OpenEHR archetypes terms to the external terminology SNOMED CT, with the capability of performing alignment at a semantic level.

The authors propose to use lexical techniques and external terminological tools in combination with context-based techniques, which use information about structural and semantic proximity to identify similarities between terms in order to establish alignments between them. The proposed approach exploits both the structural context of archetypes and the terminology context, where concepts are logically defined through the relationships (hierarchical and definitional) to other concepts. A set of 25 OBSERVATION archetypes with 477 bound terms was used to test the method. Of these,
342 terms (74.6\%) were linked with $96.1 \%$ precision, $71.7 \%$ recall and 1.23 SNOMED CT concepts on average for each mapping. It has been detected that about one third of the archetype clinical information is grouped logically. Context-based techniques take advantage of this to increase the recall and to validate a $30.4 \%$ of the bindings produced by lexical techniques.

This research demonstrates the interest in establishing the modeling of data structures on ontologies. It also shows that it is possible to semi-automatically map archetype terms to a standard terminology with a high level of precision and recall, with the help of appropriate contextual and semantic information from both models. Thus, as in other papers of this selection, contextualization is the key word in the implementation of semantic technologies in information systems.

\section{Patrick J, Li M}

\section{An ontology for clinical questions about the contents of patient notes}

\section{J Biomed Inform 2012 Apr;45(2):292-306}

The considerable amount of information available in electronic patient records make it an attractive resource for answering a variety of questions that users may have. Current information retrieval techniques have proven quite successful at locating patient records that might be relevant to a user's query. Many studies have been completed on question classification in the open domain, however only a limited amount of work has focused on the medical field. Also, to the best of our knowledge, most of these medical question classifications were designed for literature based question and answering systems..

The authors describe a four stage approach. Firstly, a relatively large set of clinical questions was collected from staff in an Intensive Care Unit. Then, a clinical question on taxonomy was designed for question and answering purposes. Subsequently an annotation guideline was created and used to annotate the question set. Finally, a multilayer classification model was built to classify the clinical questions. In this paper, a comprehensive study on clinical questions was completed. A major outcome 
of this work is the multilayer classification model. It serves as a basic component of a patient's record based on a clinical question and answering system. Also, the question collections can be reused by the research community to improve the efficiency of their own question and answering systems.

On the basis that there will always be free text available in the Electronic Health Record (EHR) system, it will subsequently be necessary to develop a natural language processing system of information retrieval in the EHR. The question of how to do this, with a $\mathrm{Q} / \mathrm{A}$ system or a semantic-based information retrieval system will arise and depend on the type of GUI institutions want to set up, as well as the context.

\section{Teodoro D, Pasche E, Gobeill J, Emonet S, Ruch P, Lovis C \\ Building a transnational biosurveillance network using semantic web technologies: requirements, design, and preliminary evaluation}

J Med Internet Res 2012 May 29;14(3):e73
Antimicrobial resistance has reached globally alarming levels and is becoming a major public health threat. Lack of effective antimicrobial resistance surveillance systems has been identified as one of the causes of increasing resistance, due to the time lag between new resistances and alerts to care providers. Several initiatives to track drug resistance evolution have been developed. However, no effective real-time and source-independent antimicrobial resistance monitoring system is available publicly. In order to propose an effective architecture that can provide real-time and source-independent antimicrobial resistance monitoring, the authors investigated the use of a Semantic Web-based model to foster integration and interoperability of inter-institutional and cross-border microbiology laboratory databases. The architecture uses an ontology-driven approach to promote the integration of a network of sentinel hospitals or laboratories. Local databases are wrapped into semantic data repositories that automatically expose local computing-formalized laboratory information on the Web. A central source mediator, based on local reasoning, coordinates the access to the semantic end points. On the user side, a user-friendly Web interface provides easy access and graphical visualization to the integrated views.

The developed prototype, the Antimicrobial Resistance Trend Monitoring System has a strong positive correlation with the European Antimicrobial Resistance Surveillance Network. The distributed monitoring architecture developed during the DebugIT European project between 2008 and 2012 can be used to build transnational antimicrobial resistance surveillance networks, and results have indicated that the Semantic Web-based approach has provided an effective and reliable solution for development of eHealth architectures.

As for the Koutkias paper, we have observed that ontology-based architecture allows proposals of systems, which integrate heterogeneous data. It in fact also offers services in the field of public health. 